\title{
Reintroduction Hazards
}

Reintroduction of a rare or endangered animal to a habitat that it once occupied is an obvious tool for the conservationist and is now for example being undertaken for the Arabian oryx. Clearly several factors have to be critically examined in the planning stage of such an operation-e.g. any vital changes in the habitat, the available food plants, the persistenceof the causes of the original extermination-but there are also many not so obvious factors that could cause failure. John Perry, of the IUCN Survival Service Commission (SSC), has listed some of them:

Each distinct native population has distinct disease antibodies. Translocated animals may lack the necessary immunities. Translocation stress may also be a factor.

Each distinct native population has distinct grazing/browsing preferences, based on available native plants. Translocated stock must adapt to a new plant community. Captive stock may have greater difficulty. Native stock may have learned to avoid local toxic plants, whereas reintroduced stock may not have done so.

Native ungulates have developed ways to protect their young from local predators; introduced stock may be unfamiliar with these.

Removal of a species brings changes in an ecosystem that may not be apparent, and may or may not favour subsequent reintroductions.

For some species there appears to be a critical mass factor in reintroductions. In the US southwest, pronghorn reintroductions have reportedly failed when fewer than 30 to 50 individuals were released. For species with a herd behaviour pattern, it seems risky to release other than a minimum typical herd with a normal age-sex distribution.

The complexities of parasites are seemingly infinite. A parasite defeated the reintroduction of elk in the Great Smokies and northern Pennsylvania; another is checking recovery of the moose in New Hampshire and Maine.

Opinions differ on the size and composition of a population necessary to provide a sufficiently diverse genetic base. Some scientists believe too narrow a base predisposes a reintroduction to ultimate failure. Yet there are numerous examples of large free-living populations arising from as few as two pairs of introduced stock.

Perry adds that these and related points are not arguments against attempted reintroductions. They do indicate that reintroductions should be made thoughtfully, not hastily, and with carefully planned monitoring, so that if a failure occurs, the reasons can be ascertained. A technique that may be appropriate is releasing animals first into an enclosure small enough to permit close observation, supplementary feeding, protection from predators, and veterinary intervention. Releasing surplus males first may disclose unsuspected hazards. Obviously one can take greater risks if stock available for reintroductions is readily available. If the species is in danger of extinction, uncontrolled releases are likely to be acceptable risks only after a first stage of controlled breeding to increase numbers.

\section{Breeding Endangered Species in San Diego}

The San Diego Zoo has established a breeding centre for endangered species, known as the Vanishing Species Reproduction Center. The project will concentrate on primates, and the aim is to breed an excess of the rarer species, such as some of the langurs and lemurs, so as to be able to supply other zoos and thus lessen the drain on wild animals. Known to the staff as the 'primate pad' it will hold up to 150 animals of 8-10 species and is right away from the areas open to the public. It will thus also give excellent facilities for behavioural research. 